\title{
Temporal evolution of a silicon surface subject to low energy ion irradiation and concurrent sample rotation
}

\author{
Tanmoy Basu ${ }^{1}$, Daniel A. Pearson ${ }^{2}$, R. Mark Bradley ${ }^{2}$ and Tapobrata Som ${ }^{1, *}$ \\ ${ }^{1}$ Institute of Physics, Sachivalaya Marg, Bhubaneswar 751 005, India \\ ${ }^{2}$ Department of Physics, Colorado State University, Fort Collins, Colorado 80523, USA
}

\begin{abstract}
We study the temporal evolution of silicon surfaces subject to low energy $\mathrm{Ar}^{+}$-ion bombardment and concurrent sample rotation. Systematic experiments are carried out in both the linear and nonlinear regimes. It is observed that an experiment which produces an anisotropic surface without sample rotation produces a statistically isotropic surface with a smaller surface roughness if the sample is rotated at a sufficiently high angular speed. Interrupted coarsening of the nanoscale mounds on the surface at long times $t$ is observed without concurrent deposition of metal impurities for the first time. We find that the characteristic lateral size and height of the mounds increase as $t^{1 / 2}$ and $t$, respectively. Both our experiments and simulations show that azimuthally rotating ripples form at a sufficiently small rotational speeds, as predicted two decades ago. Finally, predictions from theories on rotating samples subject to ion bombardment are tested.
\end{abstract}

Keywords: Low energy ion irradiation, Sputtering, Concurrent sample rotation, Surface morphology

PACS Nos. 81.05.Cy, 81.16.Rf, 61.80.Jh, 87.64.Dz

*Corresponding author; Electronic mail: tsom@,iopb.res.in 


\section{Introduction}

Ion-beam induced self-organized nanoscale pattern formation on solid surfaces has become a popular research topic in recent times. Low energy ion exposure is known for giving rise to substantial changes in surface morphology that depend on the ion species, energy, and incidence angle [1]. Oblique-incidence ion bombardment often leads to the formation of periodic height modulations (or "ripples") on the surface of an amorphous solid [2-5]. It is well known that such surface modulations are due to an interplay between curvaturedependent sputter erosion [2,6-7], mass redistribution [8] and various relaxation mechanisms (e.g. surface diffusion) occurring during ion bombardment of a solid.

Although rippled $\mathrm{Si}$ substrates are becoming increasingly important as templates for tuning magnetic and optical properties of thin films [9-11], the formation of ripples is unwanted in various circumstances, such as secondary ion mass spectroscopy (SIMS), Auger electron spectroscopy (AES), and ion milling. In the simplest kind of SIMS or AES apparatus, the primary ions are obliquely incident on the stationary surface of the sample. Thus, as sputtering proceeds, ripples can be formed, and this leads to rapid degradation of the depth resolution.

Zalar first demonstrated that this problem can often be overcome by rotating the sample with a constant angular velocity about its surface normal as the depth profiling proceeds [12]. Zalar rotation has subsequently been used by many other groups, who found that in many cases, the surface actually becomes flatter as the solid is eroded [13]. Consequently, ion sputtering with concurrent sample rotation has also been used as a means of preparing ultrasmooth surfaces for optical applications [14]. Sample rotation does not always prevent surface roughening, however. In some instances, the sample roughens while it is 
simultaneously eroded and rotated, albeit at a slower rate than it does when it is stationary $[13,15]$

A theory that describes the surface of a rotating sample that is bombarded with a broad ion beam was advanced by Bradley and Cirlin [16]. This theory applies only in the early-time, linear regime. Subsequently, Bradley [17] incorporated the leading-order nonlinear terms into the theory. The equation of motion is the isotropic Kuramoto-Sivashinsky equation to a good approximation if the rotational angular velocity $\omega$ is sufficiently high. The surface roughness asymptotes to a finite steady-state value and the topography is a disordered array of mounds in that event. In the opposite limit of slow rotation, Bradley argued that if the surface is unstable, ripples with a wavevector that rotates with angular velocity $\omega$ will be present in the steady state.

In recent years it has been discovered that ion-induced mass redistribution [8] plays an important role in nanoscale pattern formation on silicon and germanium targets, and it has been speculated that this may true of other elemental materials as well [18-20]. A number of theories of this phenomenon have been advanced [21-27], including one in which the effects of sample rotation were explored [28]. Mass redistribution was not taken into account in Refs. [16] and [17], but, as we shall see in Section II, incorporating this phenomenon into the theory is relatively straightforward.

While a few experimental studies of sample rotation during ion bombardment have been carried out [13-15,29-31], more systematic experimental work is needed to fully understand its effects and to test the applicability of the proposed theories. In the present study, we carry out careful experiments to determine the effect of concurrent sample rotation during low energy ion-beam irradiation of silicon. Silicon samples are exposed to low energy argon ions at different incidence angles. It is observed that with concurrent sample rotation, a flat surface 
is stable whenever it would also be stable in absence of any sample rotation. On the other hand, mound-like structures are formed at certain oblique incidence angles with concurrent sample rotation. In contrast, a ripple morphology develops at those angles when the sample is stationary. The linear regime of pattern formation is identified using the solid flow model $[25,32]$ and experiments are carried out in this regime at different angles of incidence with and without concurrent sample rotation. In addition, a detailed study on the temporal evolution of the surface morphology is performed at an incidence angle of $60^{\circ}$, experimentally showing the occurrence of "interrupted coarsening" $[21,28,33]$. This is the first time this phenomenon has been observed on a silicon sample in the absence of metal impurities. To explore the effect of varying the rotational speed, the samples are subjected to concurrent rotation with five different angular speeds for a particular fluence. Moreover, to look for rotating ripples with concurrent sample rotation with a low rotational speed $\omega$, atomic force microscopy (AFM) data are presented for a particular angle of incidence, namely $\theta=67^{\circ}$. Our experimental results are in good accord with the predictions made by the theories of Bradley and Muñoz-García et al. [17,28].

Our work also includes a theoretical component. After giving a theoretical overview, we carry out numerical integrations of the equation of motion proposed by Bradley [17] for various angular velocities $\omega$. We find that as $\omega$ is increased, there is a crossover from a regime in which there are azimuthally rotating ripples to one in which a disordered array of mounds forms. This is in accord with both our experiments and Bradley's prediction [17].

\section{Theoretical overview}

Consider the dynamics of the surface of an elemental solid that is bombarded with an obliquely-incident broad beam of noble gas ions. To begin, we will consider the case in which the sample is stationary. Suppose that initially the solid occupies the region $z \leq 0$, and 
that the ions' angle of incidence is $\theta$. We orient the $x$ axis so that the momenta of the incident ions are parallel to the $x-z$ plane. The surface of the solid remains flat as it is eroded with a velocity we will denote by $v_{0}$, so that the height of the surface above the point $(x, y)$ in the $x-y$ plane is $h(x, y, t)=-v_{0} t$ at time $t$.

We next consider the time evolution of the surface of the solid when its initial height is small but is not necessarily zero. The surface height is then $h=-v_{0} t+u$, where $u$ depends on $x$, $y$ and $t$. We assume that the surface height varies slowly with position. Including the lowest order nonlinearities [34], the equation of motion is

$$
u_{t}=v_{0}^{\prime} u_{x}-A_{1} u_{x x}-A_{2} u_{y y}-B \nabla^{2} \nabla^{2} u+\frac{1}{2} \lambda_{1} u_{x}^{2}+\frac{1}{2} \lambda_{2} u_{y}^{2}
$$

where the subscripts $x, y$ and $t$ denote partial derivatives. The constants $v_{0}^{\prime}, A_{1}, A_{2}, \lambda_{1}, \lambda_{2}$, and $B>0$ depend on the choice of ion beam and target material. In Eq. (1), the first term on the right-hand side is due to the slope dependence of the sputter yield, the second and third terms come from curvature-dependent sputtering and ion-induced mass redistribution, and the fourth term accounts for the smoothing effects of surface self-diffusion or for ion-induced viscous flow in a thin layer at the surface of the solid [35]. The final two terms make Eq. (1) nonlinear and have an important effect once the surface width becomes sufficiently large. Equation (1) is the anisotropic Kuramoto-Sivashinsky equation [36].

Now suppose that the solid is not stationary but is instead rotating with constant angular velocity $\omega$ about the $z$ axis. In the frame of reference that rotates with the sample, Eq. (1) still holds but with $\partial_{\mathrm{x}}$ replaced by $\cos (\varphi) \partial_{\mathrm{x}}+\sin (\varphi) \partial_{\mathrm{y}}$ and $\partial_{\mathrm{y}}$ replaced by $-\sin (\varphi) \partial_{\mathrm{x}}+\cos (\varphi) \partial_{\mathrm{y}}$, where $\varphi \equiv \omega t$. Applying these transformations to Eq. (1) yields 


$$
\begin{aligned}
& u_{t}=v_{0}^{\prime}\left(u_{x} \cos \varphi+u_{y} \sin \varphi\right)-A \nabla^{2} u-B \nabla^{2} \nabla^{2} u+\frac{1}{2} \lambda|\nabla u|^{2}-\frac{1}{2} \Delta A \cos (2 \varphi)\left(u_{x x}-u_{y y}\right) \\
& -\Delta A \sin (2 \varphi) u_{x y}+\frac{1}{4} \Delta \lambda \cos (2 \varphi)\left(u_{x}^{2}-u_{y}^{2}\right)+\frac{1}{2} \Delta \lambda \sin (2 \varphi) u_{x} u_{y}
\end{aligned}
$$

where $A \equiv \frac{1}{2}\left(A_{1}+A_{2}\right), \lambda \equiv \frac{1}{2}\left(\lambda_{1}+\lambda_{2}\right), \Delta A \equiv A_{1}-A_{2}$, and $\Delta \lambda \equiv \lambda_{1}-\lambda_{2}$. The terms proportional to $v_{0}^{\prime}$ can be eliminated by transforming to a reference frame with an origin that moves in a circle and that has axes parallel to the $x$ and $y$ axes. In particular, we set

$$
\begin{aligned}
\tilde{x} & \equiv x+v_{0}^{\prime} \sin (\varphi) / \omega, \\
\widetilde{y} & \equiv y-v_{0}^{\prime} \cos (\varphi) / \omega, \\
\widetilde{t} & \equiv t
\end{aligned}
$$

and drop the tildes. This yields the equation of motion

$$
\begin{aligned}
& u_{t}=-A \nabla^{2} u-B \nabla^{2} \nabla^{2} u+\frac{1}{2} \lambda|\nabla u|^{2}-\frac{1}{2} \Delta A \cos (2 \omega t)\left(u_{x x}-u_{y y}\right) \\
& -\Delta A \sin (2 \omega t) u_{x y}+\frac{1}{4} \Delta \lambda \cos (2 \omega t)\left(u_{x}^{2}-u_{y}^{2}\right)+\frac{1}{2} \Delta \lambda \sin (2 \omega t) u_{x} u_{y} .
\end{aligned}
$$

Equation (2) will be integrated numerically in Sec. $4 \mathrm{E}$ to illustrate the differing behaviour of the surface for low and high angular velocities.

For the nonrotating case, we know from the Bradley-Harper theory [2] that

1. For $A_{1}<0$ and $A_{2}<0$, the surface becomes flat.

2. For $A_{1}>0$ and $A_{1}>A_{2}$, parallel-mode ripples emerge in the linear regime.

3. For $A_{2}>0$ and $A_{2}>A_{1}$, perpendicular-mode ripples emerge in the linear regime.

If the sample is rotating and its angular velocity $\omega$ is large compared to the linear growth rate $|A|^{2} / 4 B$, on the other hand, Eq. (2) may be replaced by its temporal average

$$
u_{t}=-A \nabla^{2} u-B \nabla^{2} \nabla^{2} u+\frac{1}{2} \lambda(\nabla u)^{2}
$$


as discussed in Ref. [17]. If $\mathrm{A}<0$, the surface becomes flat. On the other hand, if $\mathrm{A}>0$, Eq. (3) is the isotropic Kuramoto-Sivashinsky equation [37,38] and the nominally flat initial surface is unstable. At sufficiently long times, the surface consists of an irregular array of mounds and it exhibits spatio-temporal chaos. The characteristic width and height of the mounds are $\sqrt{B / A}$ and $A /|\lambda|$, respectively.

At sufficiently early times, the nonlinear terms in Eqs. (1) and (3) can be neglected. Without sample rotation, the growth rate of the linearly selected mode, $\sigma_{n r}$, is given by

$$
\sigma_{n r}=\left|A_{1}\right|^{2} / 4 B \quad \text { for } A_{1}>0 \text { and } A_{1}>A_{2},
$$

and by

$$
\sigma_{n r}=\left|A_{2}\right|^{2} / 4 B \quad \text { for } A_{2}>0 \text { and } A_{2}>A_{1} \text {. }
$$

On the other hand, with concurrent sample rotation at a high angular velocity, the growth rate of the linearly selected mode is

$$
\sigma_{r}=|A|^{2} / 4 B \quad \text { for } A>0 .
$$

A straightforward analysis now shows that if the surface is unstable when the sample is stationary, then

$$
\sigma_{n r} \geq \sigma_{r}
$$

Physically, this means that amplitude of the mounds formed with rapid sample rotation grows more slowly than the amplitude of the ripples formed without sample rotation. It is also possible for $A=\left(A_{1}+A_{2}\right) / 2$ to be negative if $A_{1}$ or $A_{2}$ is positive. In that event, the surface develops ripples without sample rotation but becomes flat if the sample is rotated rapidly.

If one appends the next-to-lowest order nonlinearity to the equation of motion, then Eq. (3) is replaced by 


$$
u_{t}=-A \nabla^{2} u-B \nabla^{2} \nabla^{2} u+\frac{1}{2} \lambda(\nabla u)^{2}+\frac{1}{2} \mu \nabla^{2}(\nabla u)^{2}
$$

where $\mu$ is a constant. This additional nonlinearity is discussed by Muñoz-García et al. [28]. Their simulations of Eq. (8) reveal that once mounds form, they coarsen for a time but then stop growing. This phenomenon is called "interrupted coarsening." The characteristic mound lateral dimension $L$ and height $H$ grow as powers of the time $t$ after the linear regime has ended but before the coarsening has been interrupted. The corresponding scaling exponents $a$ and $b$, which will be defined by the relations $L \sim t^{a}$ and $H \sim t^{b}$, are presently unknown. At very long times, a crossover occurs and $L$ and $H$ tend to steady-state values.

If the sample is rotated slowly enough that $\omega \ll|A|^{2} / 4 B$, in the steady state ripples whose wavevector rotates with angular velocity $\omega$ will be present on the solid surface [17]. It is not apparent whether there will be a phase lag between the projected direction of ion incidence and the dominant ripple wavevector or whether the two will rotate in unison. This issue will be investigated in the simulations we will discuss in Sec. 4 E.

\section{Experimental}

A $p$-type $\mathrm{Si}(100)$ wafer (B-doped, resistivity $0.01-0.02 \Omega$-cm) was sliced into $1 \mathrm{~cm} \times 1 \mathrm{~cm}$ pieces for the present study. All irradiation experiments were carried out in a UHVcompatible experimental chamber (Prevac, Poland) which was equipped with a 5-axis sample manipulator and a low energy (up to $2 \mathrm{keV}$ ) electron cyclotron resonance (ECR) based broad beam, filamentless ion source (Tectra GmbH, Germany). The chamber's base pressure was below $5 \times 10^{-9}$ mbar and the working pressure was maintained at $3 \times 10^{-4}$ mbar with the help of a differential pumping unit. Two different argon ion energies were chosen (viz. 500 and 750 $\mathrm{eV})$ to perform a thorough angle dependent $\left(\theta=0^{0}-85^{\circ}\right)$ study of the evolution of silicon surface topography with and without concurrent azimuthal sample rotation (for a rotational 
speed of $1 \mathrm{rpm})$. However, because our findings for these two ion energies are quite similar, we will focus on the results for an ion energy of $500 \mathrm{eV}$. In addition, systematic studies were carried out at two higher incidence angles of $60^{\circ}$ and $67^{\circ}$ for rotational speeds of $0.08 \mathrm{rpm}$ and $1 \mathrm{rpm}$ and without concurrent sample rotation. For the present study, the beam diameter was measured to be $3 \mathrm{~cm}$ and a constant current density of $21.4 \mu \mathrm{A} \mathrm{cm}^{-2}$ was used. This led to an ion flux of $1.3 \times 10^{14}$ ions $\mathrm{cm}^{-2} \mathrm{~s}^{-1}$. Most of the experiments were performed at a fixed fluence of $5 \times 10^{17}$ ions $\mathrm{cm}^{-2}$ at six different angular speeds between 0.08 and $1 \mathrm{rpm}$. In addition, measurements were carried out to study the fluence dependence (in the range of $1 \times 10^{16}$ to $1 \times 10^{18}$ ions $\mathrm{cm}^{-2}$ ) and the temporal evolution of the surface topography at different incidence angles both with and without concurrent sample rotation.

\section{Results and discussion}

\section{A. Evolution of surface topography with concurrent sample rotation}

Examples of the evolution of the surface topography at different incidence angles with concurrent sample rotation are depicted in Figs. 1(a)-(f). Figure 1(a) shows the evolution of a nearly smooth initial surface $(\mathrm{rms}$ roughness $=0.1 \mathrm{~nm}$ ) of an ion-exposed silicon sample with $\theta=40^{0}$ and at a fluence of $5 \times 10^{17}$ ions $\mathrm{cm}^{-2}$ with continuous azimuthal rotation at the angular velocity $\omega=0.08 \mathrm{rpm}$. Figs. $1(\mathrm{~b})-(\mathrm{d})$ show nanoscale mound formation at the angles of incidence $60^{\circ}, 67^{\circ}$ and $70^{\circ}$, respectively. Our previous experiments without any sample rotation showed the presence of ripples and mounds at these angles for the fluence chosen here [39]. It is interesting to note that Figure 1(e) reveals the presence of the biggest mounds (with an average diameter of approximately $80 \mathrm{~nm}$ ) for the angle of incidence $\theta=72.5^{\circ}$, whereas a smooth surface is observed after ion bombardment at $\theta=85^{\circ}$ [Fig. 1(f)] with concurrent sample rotation. Thus, it is observed that with concurrent sample rotation a smooth surface develops (at both ion energies) up to an angle of incidence of $51^{\circ}$ and for near 
grazing incidence $\left(\theta>77.5^{\circ}\right)$, whereas at intermediate angles, mounds develop on the surface. In this process, a spatially anisotropic surface is transformed into a statistically isotropic one. This is seen from the insets in Figs. 1(b)-(e), since the respective 2D FFTs show the absence of any special direction.

The corresponding surface roughness plots are shown in Fig. 2 as a function of the ion incidence angle with and without sample rotation. Interestingly, this figure reveals that concurrent sample rotation can cause a significant reduction in the rms roughness for angles of incidence which lead to mound formation.

The mound size is observed to increase with angle of incidence until a critical angle is reached. For $\theta=60^{\circ}, 67^{\circ}$, and $70^{\circ}$, the average lateral dimension of the mounds are 28,32 , and $38 \mathrm{~nm}$, respectively. At higher angles of incidence, the lateral dimension of the mounds decreases, until, as is evident from Fig. 1(f), the surface remains flat for $\theta=85^{\circ}$.

At first sight, Fig. 2 seems to agree with the theoretical prediction of Eq. (7). However, this is only the case if all of the data were taken in the early-time, linear regime in which Eq. (7) applies. Therefore, care must be taken to insure that this is the case before we can conclude that experimental evidence for the validity of Eq. (7) has been garnered.

In the early-time, linear regime, Eq. (7) applies. To test this prediction, we have performed angle-dependent experiments by selecting a proper timescale in which the pattern formation can be described by the linear approximation. To find this timescale, we have invoked the solid flow model and calculated the upper limit of the linear regime of pattern evolution $[25,32]$. In this framework, for the angular window of $\theta=55^{\circ}-67^{\circ}$, an erosion time of $300 \mathrm{~s}$ can be considered to be a safe enough time scale to remain within the linear regime, whereas $120 \mathrm{~s}$ is appropriate for the angular window of $\theta=70^{\circ}-72.5^{\circ}$. Likewise, we have identified the 
linear regime over the entire angular window of $\theta=0^{\circ}-85^{\circ}$ and carried out systematic experimentation with and without concurrent sample rotation. For this angular window, we have chosen the rotational speed to be $1 \mathrm{rpm}$ ( or $0.104 \mathrm{rad} \mathrm{s}^{-1}$ ) so that we can safely use the linear form of Eq. (3).

In Fig. 3, we combine our observations into a parametric phase diagram (energy versus ion incidence angle) under two experimental conditions: with and without concurrent sample rotation. To the best of our knowledge, this is the first study which addresses the temporal evolution of a silicon surface at various ion energies for both static and rotating samples. From this phase diagram, it is clear that under both conditions, the Si surface remains stable up to $\theta=51^{\circ}$ and beyond $77.5^{\circ}$, whereas parallel-mode ripples undergo a transition to mounds with concurrent sample rotation. Notice as well that the behaviour is not discernibly different for the two ion energies we studied. This provides support for our choice to focus on the results for $500 \mathrm{eV}$ argon ions.

Figure 4 shows the roughness versus the angle of incidence $\left(\theta=55^{0}-72.5^{\circ}\right)$ for $500 \mathrm{eV}$ argon ions in the linear regime. One observes that the rms roughness decreases when the concurrent rotation is switched on. The reason for choosing this smaller angular window stems from the fact that bombarding at these angles of incidence produced the most prominent ripples. The results clearly demonstrate the validity of the prediction given by Eq. (7).

\section{B. Temporal evolution of mounds}

The temporal evolution of the mounds with concurrent sample rotation is shown in Fig. 5 for $\theta=67^{0}$. Fig. 5(a) shows the morphology corresponding to an exposure time of $660 \mathrm{~s}$, when small mounds are visible. The characteristic width of the mounds grows with time for exposure times between 660 and $6600 \mathrm{~s}$. 
The time evolution of the mounds is quantified in Figs. 6 (a) and (b), where we observe that the average mound width $L$ is proportional to the square root of the erosion time. This is accompanied by a linear increase in the rms roughness $w$ with the erosion time. We therefore obtain estimates for the scaling exponents defined in Section $2: a \cong 0.5$ and $b \cong 1$. Furthermore, the mounds are isotropic, as can be seen from the insets of Figs. 5(a)-(d); it is clear that the respective 2D FFTs do not show any significant anisotropy.

In the linear regime, the rms roughness $w$ increases exponentially and the average mound width $L$ is approximately constant. This early-time behaviour does not appear in Figures 6 and 7 because nonlinear effects had already become important for the earliest times shown. The Kuramoto-Sivashinsky equation (3) does not yield a regime in which the mounds coarsen with time, and so is not adequate to explain our experimental results. Equation (8), however, represents a more refined equation of motion that does give mound coarsening. This coarsening is ultimately interrupted, however. This raises a question: can interrupted coarsening be observed in our experiments?

To observe interrupted coarsening for the angle of incidence $\theta=67^{\circ}$, very high fluences would be necessary. We therefore studied the temporal evolution of the mounds for $\theta=60^{\circ}$. The results, which are shown in Fig. 7, very clearly show that the coarsening is interrupted after sufficient time has passed, as predicted by Muñoz-García et al. [28]. The coarsening is interrupted for $\theta=60^{\circ}$ for an irradiation time that is less than $3.0 \times 10^{3} \mathrm{~s}$. By contrast, the mounds continued to grow in size at this time for $\theta=67^{\circ}$. This shows that interrupted coarsening takes place sooner for the angle of incidence $\theta=60^{\circ}$ than for $\theta=67^{\circ}$.

Interrupted coarsening has previously been observed only in experiments in which metal impurities were deposited during ion erosion [33], even though it has been predicted to occur in the absence impurity co-deposition [28]. It is known that metal impurities can have a decisive influence on the nanoscale patterns that develop --- for example, they can destabilize 
a surface that would otherwise remain flat [40]. Our results provide the first experimental evidence that supports the prediction that interrupted coarsening can occur without concurrent deposition of a second atomic species.

\section{Dependence on angular velocity}

The effect of the angular velocity on the formation of the nanostructures has not been studied to a great extent [31]. To explore the consequences of changing the angular velocity, we exposed silicon samples to a $500 \mathrm{eV}$ argon ion beam at the angle of incidence $\theta=67^{\circ}$ and varied the angular velocity over the range of $0.08-1 \mathrm{rpm}\left(8 \times 10^{-3}\right.$ to $\left.44 \times 10^{-3} \mathrm{rad} \mathrm{s}^{-1}\right)$ at a constant fluence of $5 \times 10^{17}$ ions $\mathrm{cm}^{-2}$. Figure 8 shows selected AFM images that illustrate the morphological evolution of these samples. The insets show the corresponding 2D FFTs. These demonstrate the statistically isotropic nature of the surfaces.

A plot of roughness as a function of the angular velocity $\omega$ is shown in Fig. 9(a), while Figs. 9(b) and (c) depict variations in the average lateral dimension of the mounds and the average mound height as a function of rotational speed, respectively. It is observed that with increasing $\omega$, the roughness and mound height both show an increasing trend up to a rotational speed of roughly $0.34 \mathrm{rpm}\left(34.8 \times 10^{-3} \mathrm{rad} \mathrm{s}^{-1}\right)$ beyond which they saturate, whereas the average lateral dimension of the mounds remains more or less constant [Fig. 9 (b)]. Recently, Chowdhury et al. have observed non-monotonic behaviour in the rms roughness over a wide range of $\omega(0-25 \mathrm{rpm})$ [31]. This behaviour is in qualitative agreement with Monte Carlo simulations modelling the sputtering of a continuously rotating sample [41]. 


\section{Existence of rotating ripples}

For a fixed angle of incidence $\theta=67^{\circ}$ and the irradiation time $300 \mathrm{~s}$, ripples whose wavevector rotated in unison with the projected ion beam direction were observed for an angular velocity of $0.08 \mathrm{rpm}$ [Fig. 10 (b)]. The FFT of the surface [shown in the inset in Fig. 10 (b)] makes the anisotropy of the surface pattern particularly apparent. This type of azimuthal rotation of ripples was predicted by Bradley [17] for low angular velocities, although its experimental verification remained hitherto unexplored. For an increased rotational speed (viz. $1 \mathrm{rpm}$ ), on the other hand, the presence of mounds was observed [Fig. 10(c)]. The surface was statistically isotropic in this case. This transition from rotating ripples to mounds was also predicted by Bradley.

\section{E. Simulations}

In order to test whether Eq. (2) reproduces the previously discussed experimentally observed phenomena, we integrated the equation numerically for various angular velocities. The integrations were performed on the domain $\{(x, y) \mid-50 \leq x, y \leq 50\}$ using 256x256 equally spaced grid points. Periodic boundary conditions were employed. In Fig. 11, we see a sequence of snapshots of the surface height obtained by simulating Eq. (2) with $A=\lambda=1$, $\Delta A=2.2, \Delta \lambda=-1 / 2$, and $\omega=0.01$. A simulation using these parameters but with $\omega=0$ produces parallel mode ripples, as is the case in Figure 10 (a). It is evident in Figure 11 that the surface has developed ripples which rotate as time passes. This is consistent with the experimental result seen in Figure 10 (b) that shows the presence of rotated ripples for sufficiently small angular velocities. 
The ripples seen in Fig. 11 rotate with the beam and without any significant phase lag. This is shown by Fig. 12, which gives the time dependence of the beam's azimuthal angle and the angle that the predominant ripple wavevector makes with the $x$-axis.

The simulations also reproduce the transition from parallel mode ripples to isotropic mounds that occurs as the angular velocity is increased. Figure 13 shows the surface height obtained from a simulation with the same parameters as used in Fig. 11, but with the higher angular velocity $\omega=2$. This transition has also been observed in kinetic Monte Carlo simulations [41,42]. In contrast to the Monte Carlo simulations of Yang et al. [42], however, we find no evidence for the formation disordered square arrays of mounds in either our simulations or our experiments. It is therefore possible that the square arrays observed by Yang et al. were simply an artefact of carrying out simulations on a simple cubic lattice.

Although Eq. (2) does reproduce some of the experimental observations, it does not yield coarsening. Equation (8), which includes the next-to-lowest order nonlinearity, does give interrupted coarsening [28], as observed in our experiments.

\section{Conclusions}

In summary, we have investigated the evolution of silicon surfaces due to low energy $\mathrm{Ar}^{+}$-ion bombardment with concurrent sample rotation. A systematic study of the dependence of the patterns on the ion energy and the angular velocity of the sample was carried out. Based on the solid flow model, a clear demarcation between linear and non-linear regimes was identified for rotating samples. The results were summarized in a phase diagram. The effect of sample rotation on the roughness evolution was explored, yielding exciting results. In particular, we observed interrupted coarsening of nanostructures with concurrent sample rotation. This had previously been seen in only in experiments in which metal impurities 
were deposited during ion erosion [33]. In addition, the scaling behaviour that occurs after the early-time linear regime but before the long-time steady state is reached was studied for the first time. We found that the characteristic lateral size and height of the mounds increase roughly as $t^{1 / 2}$ and $t$, respectively. Another novelty of the present work is to provide experimental evidence of the existence of sample rotation speeds low enough that azimuthally rotating ripples can be observed. As we showed using numerical integrations, the equation of motion proposed by Bradley [17] predicts that this phenomenon will occur. Our experimental studies indicate that the average lateral size of the mounds is relatively insensitive to small variations in the rotational speed, while the surface roughness and the height of the mounds grow and eventually saturate when viewed as functions of that variable. A number of our experimental results agree well with the theoretical predictions made by Bradley [17] and Muñoz-García et al. [28].

The experiments, analytical theory, and simulations reported here will be important for applications in the field of ion-beam shaping of surface nanostructures and roughness engineering. For simplicity, the research in this paper is focused entirely on an elemental target material (silicon). Future studies of the effects of ion bombardment on rotating binary materials are planned; past experiments $[29,20]$ and recent theoretical work [43] suggest that new and interesting phenomena may be observed.

\section{Acknowledgments}

The authors would like to thank Mohit Kumar, Institute of Physics, Bhubaneswar for his help during the course of this work. R.M.B. thanks the National Science Foundation for its support through grant DMR-1305449. 


\section{References}

[1] T. Som and D. Kanjilal (Eds.), Nanofabrication by Ion-beam Sputtering: Fundamentals and Applications, Pan Stanford Publishing, 2012

[2] R. M. Bradley and J. M. E. Harper, J. Vac. Sci. Technol. A 6 (1988) 2390

[3] K. Elst and W. Vandervorst, J. Vac. Sci. Technol. A 12 (1994) 3205.

[4] S. W. MacLaren, J. E. Baker, N. L. Finnegan, and C. M. Loxton, J. Vac. Sci. Technol. A 10 (1992) 468.

[5] R. M. Bradley and J. M. E. Harper, Defect Diffusion Forum 61 (1988) 55.

[6] E. Chason, T. M. Mayer, B. K. Kellerman, D. T. McIlroy, and A. J. Howard, Phys. Rev. Lett. 72 (1994) 3040.

[7] T. M. Mayer, E. Chason, and A. J. Howard, J. Appl. Phys. 76 (1994) 1633.

[8] G. Carter and V. Vishnyakov, Phys. Rev. B 54 (1996) 17647.

[9] M. Ranjan, T. W. H. Oates, S. Facsko, and W. Möller, Opt. Lett. 35 (2010) 2576; T. W.

H. Oates, M. Ranjan, S. Facsko, and H. Arwin, Opt. Exp. 19 (2011) 3.

[10] T. Basu, Mohit Kumar, S. Nandy, B. Satapati, C. P. Saini, A. Kanjilal, and T. Som, J. Appl. Phys. 118 (2015) 104903.

[11] M. O. Liedke, M. Körner, K. Lenz, F. Grossmann, S. Facsko, and J. Fassbender, Appl. Phys. Lett. 100 (2012) 242405.

[12] A. Zalar, Thin Solid Films 124 (1985) 223.

[13] E.-H. Cirlin, J. J. Vajo, R. E. Doty, and T. C. Hasenberg, J. Vac. Sci. Technol. A 9 (1991) 1395.

[14] F. Frost, B. Ziberi, A. Schindler and B. Rauschenbach, Appl. Phys. A 91 (2008) 551.

[15] E.-H. Cirlin, J. J. Vajo, and T. C. Hasenberg, J. Vac. Sci. Technol. B 12 (1994) 269.

[16] R. M. Bradley and E.-H. Cirlin, Appl. Phys. Lett. 68 (1996) 3722.

[17] R. M. Bradley, Phys. Rev. E 54 (1996) 6149. 
[18] C. S. Madi, E. Anzenberg, K. F. Ludwig, Jr., and M. J. Aziz, Phys. Rev. Lett. 106 (2011) 066101.

[19] S. A. Norris, J. Samela, L. Bukonte, M. Backman, F. Djurabekova, K. Nordlund, C. S. Madi, M. P. Brenner, and M. J. Aziz, Nature Commun. 2 (2011) 276.

[20] E. Anzenberg, J. C. Perkinson, C. S. Madi, M. J. Aziz and K. F. Ludwig, Phys. Rev. B 86 (2012) 245412.

[21] M. Castro, R. Cuerno, L. Vázquez, and R. Gago, Phys. Rev. Lett. 94 (2005) 016102.

[22] J. Muñoz-García, M. Castro, and R. Cuerno, Phys. Rev. Lett. 96 (2006) 086101.

[23] J. Muñoz-García, R. Cuerno, and M. Castro, Phys. Rev. B 78 (2008) 205408.

[24] M. Castro and R. Cuerno, Appl. Surf. Sci. 258 (2012) 4171.

[25] M. Castro, R. Gago, L. Vázquez, J. Muñoz-García and R. Cuerno, Phys. Rev. B 86 (2012) 214107.

[26] S. A. Norris, Phys. Rev. B 85 (2012) 155325.

[27] S. A. Norris, Phys. Rev. B 86 (2012) 235405.

[28] J. Muñoz-García, R. Cuerno, and M. Castro, J. Phys. Condens. Matter 21 (2009) 224020.

[29] F. Frost, A. Schindler, and F. Bigl, Phys. Rev. Lett. 85 (2000) 4116.

[30] F. Frost, B. Ziberi, T. Hoche and B. Rauschenbach, Nucl. Inst. Meth. Phys. Res. B 216 (2004) 9.

[31] D. Chowdhury, D. Ghose, and B. Satpati, Mater. Sci. Eng. B 179 (2014) 1.

[32] T. Basu and T. Som, Appl. Surf. Sci. 310 (2014) 142.

[33] J. Muñoz-García, R. Gago, L. Vázquez, J. A. Sánchez-García, and R. Cuerno, Phys. Rev. Lett. 104 (2010) 026101.

[34] J. Muñoz-García, L. Vázquez, M. Castro, R. Gago, A. Redondo-Cubero, A. MorenoBarrado and R. Cuerno, Mater. Sci. Eng. R-Rep. 86 (2014) 1.

[35] C. C. Umbach, R. L. Headrick, and K.-C. Chang, Phys. Rev. Lett. 87 (2001) 246104. 
[36] M. Rost and J. Krug, Phys. Rev. Lett. 75 (1995) 3894.

[37] Y. Kuramoto and T. Tsuzuki, Prog. Theor. Phys. 55 (1976) 356.

[38] G. I. Sivashinsky, Acta Astronaut. 4 (1976) 1177.

[39] T. Basu, J. R. Mohanty, T. Som, Appl. Surf. Sci. 258 (2012) 9944.

[40] G. Ozaydin, A. S. Ozcan, Y. Wang, K. F. Ludwig, H. Zhou, R. L. Headrick, and D. P. Siddons, Appl. Phys. Lett. 87 (2005) 163104; G. Ozaydin-Ince, K. F. Ludwig, Jr., H. Zhou, and R. L. Headrick, J. Vac. Sci. Technol. B 26 (2008) 551; J. Zhou, S. Facsko, M. Lu, and W. Moller, J. Appl. Phys. 109 (2011) 104315; R. M. Bradley, Phys. Rev. B 83 (2011) 195410; R. M. Bradley, Phys. Rev. B 85 (2012) 115419; R. M. Bradley, Phys. Rev. B 87 (2013) 205408. [41] T. Yasseri and R. Kree, Nucl. Instrum. Meth. Phys. Res. B 268 (2010) 2496.

[42] Z. Yang, M. A. Lively and J. P. Allain, Phys. Rev. B 91 (2015) 075427.

[43] M. P. Harrison and R. M. Bradley, J. Phys.: Cond. Matt. 27 (2015) 295301. 


\section{Figure captions:}

Figure 1 (Colour online): Representative AFM images of a rotating Si surface bombarded with a 500 eVAr ion beam at different incidence angles $\left(\theta=40^{\circ}-85^{\circ}\right)$ and to a fluence of $5 \times 10^{17}$ ions $\mathrm{cm}^{-2}$. The height scales in (a)-(f) are $1 \mathrm{~nm}, 4 \mathrm{~nm}, 8 \mathrm{~nm}, 11 \mathrm{~nm}, 23 \mathrm{~nm}$, and $2 \mathrm{~nm}$, respectively. The insets in (a)-(f) show 2D FFTs obtained from the corresponding images.

Figure 2 (Colour online): The rms roughness plotted as a function of ion incidence angle for an ion fluence of $5 \times 10^{17}$ ions $\mathrm{cm}^{-2}$. The solid lines are guides to the eye.

Figure 3 (Colour online): Energy-angle parametric phase diagrams, showing the evolution of different patterns with and without concurrent sample rotation for a rotational speed of 1 rpm. All data were taken in the linear regime for the angle of incidence in question. For clarity, we have displayed data for only two ion energies, although the remaining data follow the same trends.

Figure 4 (Colour online): Roughness plots in the linear regime as a function of ion incidence angle. The plots are for $500 \mathrm{eV}$ Ar-ions. The solid lines are guide to the eyes.

Figure 5 (Colour online): AFM images showing the temporal evolution of the mounds for different irradiation times, viz. (a) $660 \mathrm{~s}$, (b) $1320 \mathrm{~s}$, (c) $3300 \mathrm{~s}$, and (d) $6600 \mathrm{~s}$. The angular velocity was $0.08 \mathrm{rpm}$ and the incidence angle was $\theta=67^{\circ}$. The height scales in (a)-(d) are 6 $\mathrm{nm}, 8 \mathrm{~nm}, 13 \mathrm{~nm}$, and $18 \mathrm{~nm}$, respectively. The insets show the 2D FFTs obtained from the corresponding images.

Figure 6 (Colour online): (a) The lateral dimension of the mounds as a function of square root of the erosion time and (b) the rms roughness as a function of erosion time for $\theta=67^{\circ}$. The dashed lines are guides to the eye. 
Figure 7 (Colour online): (a) The average lateral dimension of the mounds as a function of square root of the erosion time and (b) the rms roughness as a function of erosion time for $\theta=60^{\circ}$. The dashed lines are guides to the eyes.

Figure 8 (Colour online): Representative AFM images that show the effect of varying the angular velocity at an incidence angle of $\theta=67^{0}$. (a) $0.17 \mathrm{rpm}\left(17.4 \times 10^{-3} \mathrm{rad} \mathrm{s}^{-1}\right)$, (b) $0.26 \mathrm{rpm}$ $\left(26.2 \times 10^{-3} \mathrm{rad} \mathrm{s}^{-1}\right)$, and (c) $0.43 \mathrm{rpm}\left(43.6 \times 10^{-3} \mathrm{rad} \mathrm{s}^{-1}\right)$. The height scale is $11 \mathrm{~nm}$. The insets show the corresponding 2D FFTs.

Figure 9 (Colour online): The variation in rms roughness with rotational speed for $\theta=67^{0}$ and at a fixed fluence of $5 \times 10^{17}$ ions $\mathrm{cm}^{-2}$. Panels (b) and (c) show plots of the average lateral dimension of the mounds and the mound height versus rotational speed, respectively.

Figure 10 (Colour online): AFM images of Si samples exposed to Ar-ion irradiation for 300 s at an incidence angle of $67^{\circ}$ : (a) without sample rotation, (b) with a sample rotation speed of $0.08 \mathrm{rpm}$, and (c) with a sample rotational speed of $1 \mathrm{rpm}$. The corresponding height scales in (a)-(c) are $5.8 \mathrm{~nm}, 2.7 \mathrm{~nm}$, and $5.3 \mathrm{~nm}$, respectively. The insets are the corresponding FFTs and the red arrow in (a) shows the direction of incident ion beam.

Figure 11 (Colour online): Snapshots of the surface height at times (a) $t=900$, (b) $t=950$, and (c) $t=1000$ obtained by simulating Eq. (2) with $A=\lambda=1, \Delta A=2.2, \Delta \lambda=-1 / 2$, and $\omega=0.01$. The snapshots are taken in the frame of the sample and the blue arrows show the projected direction of the ion beam.

Figure 12 (Colour online): The blue line shows the beam's azimuthal angle $\phi$ versus time. The orange curve shows the azimuthal angle of the wavevector of the most highly excited Fourier mode. The simulation is the same as was used to produce Figure 11. 
Figure 13 (Colour online): Snapshot at time $t=1000$ of the surface height obtained by simulating Eq. (2) with $A=\lambda=1, \Delta A=2.2, \Delta \lambda=-1 / 2$, and $\omega=2$. 

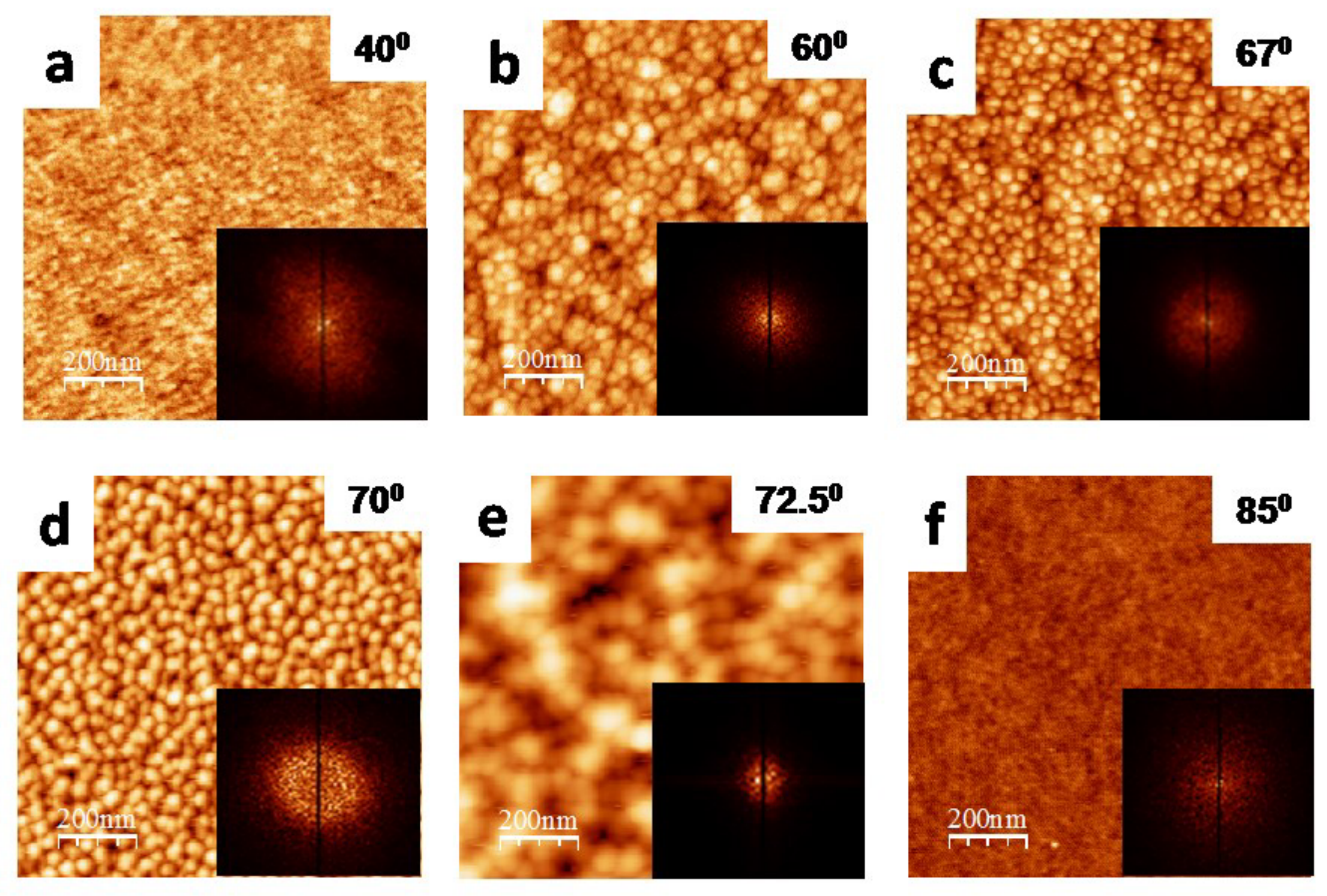

Basu et al.

Figure 1 


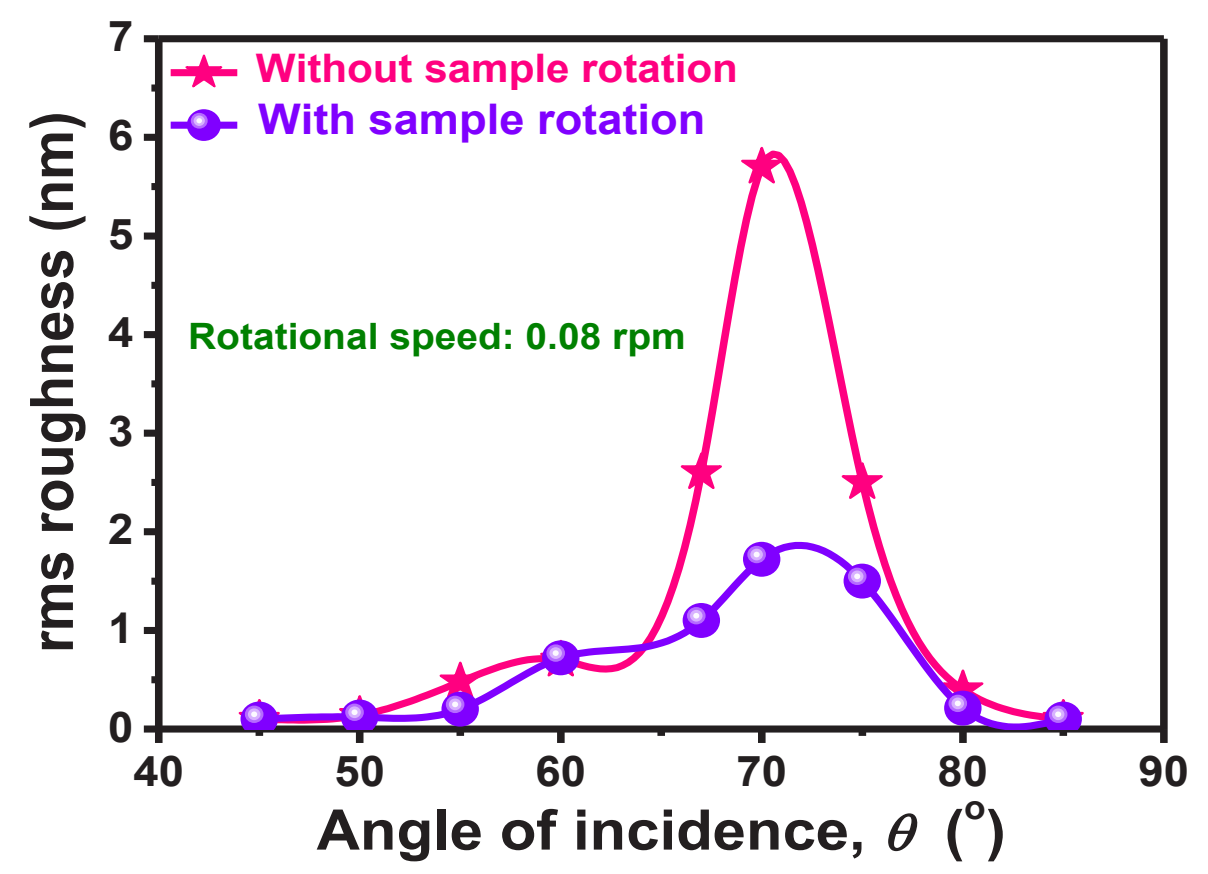

Basu et al.

Figure 2 


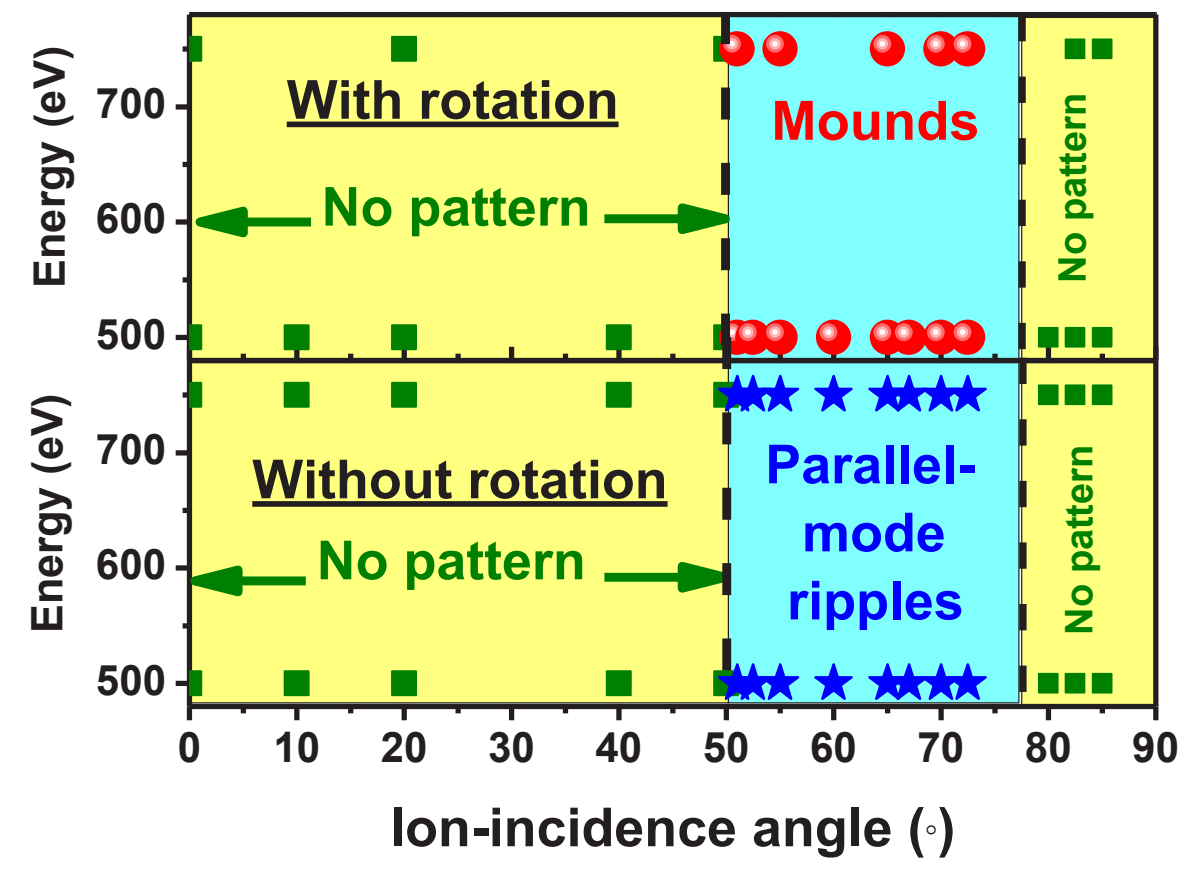

Basu et al.

Figure 3 


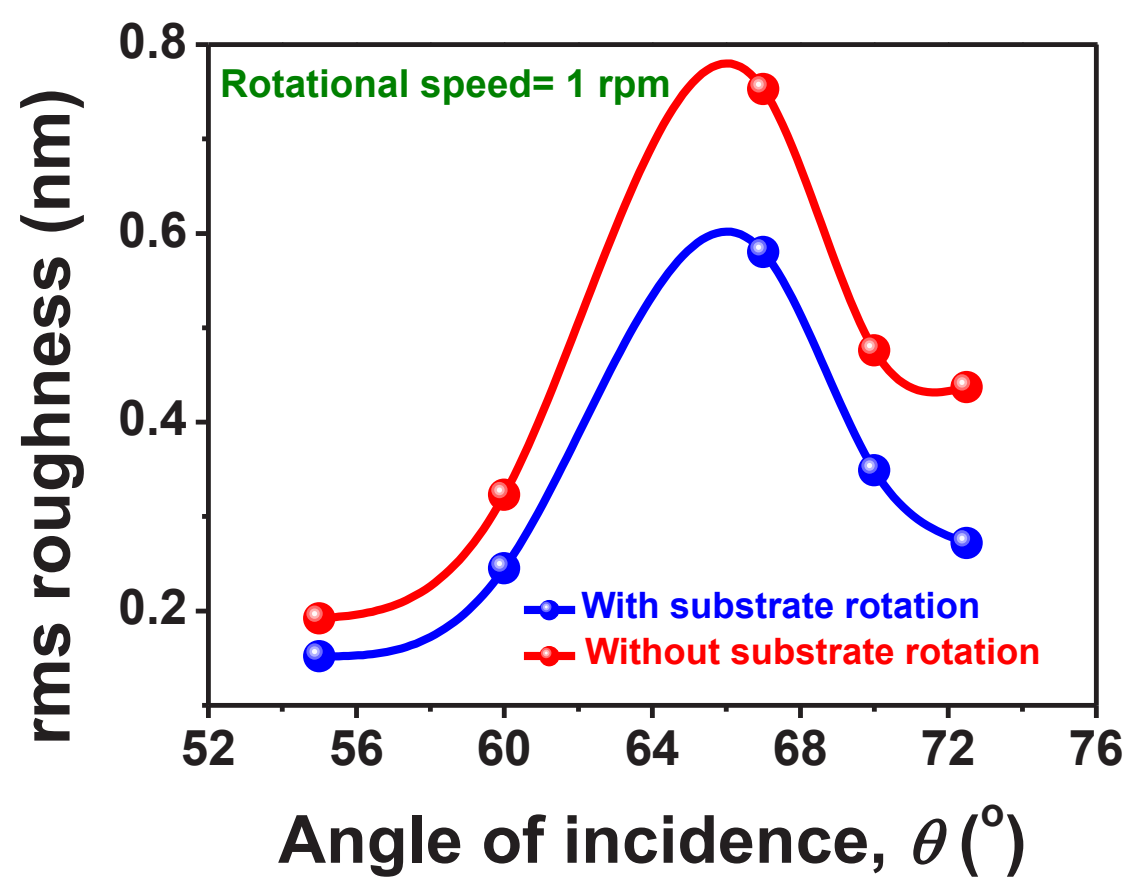

Basu et al.

Figure 4 

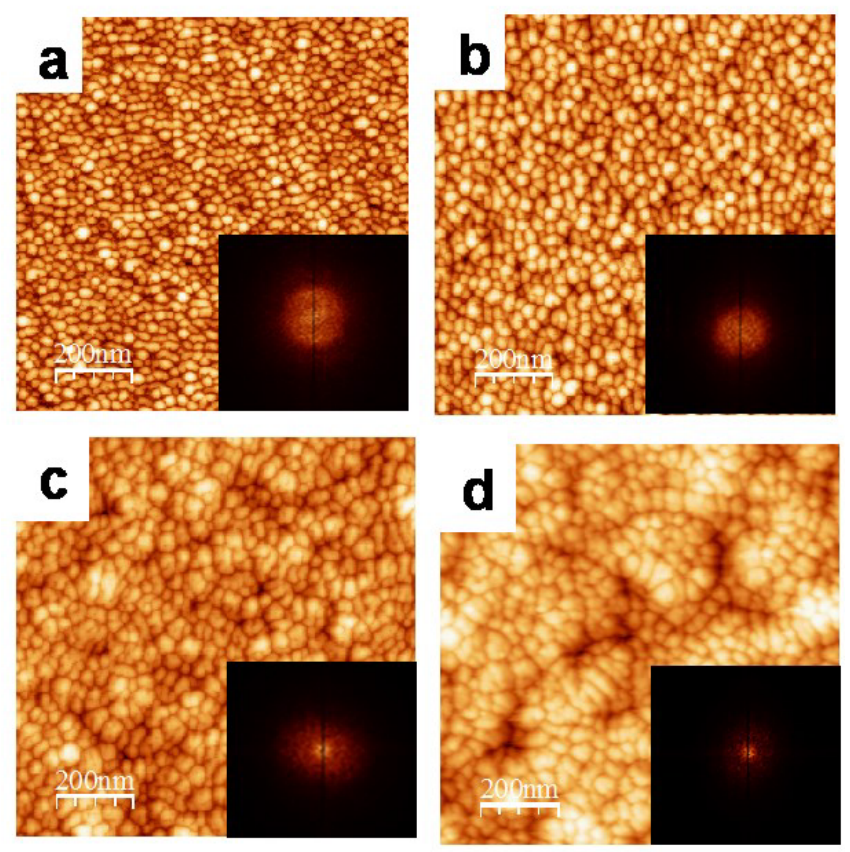

Basu et al.

Figure 5 

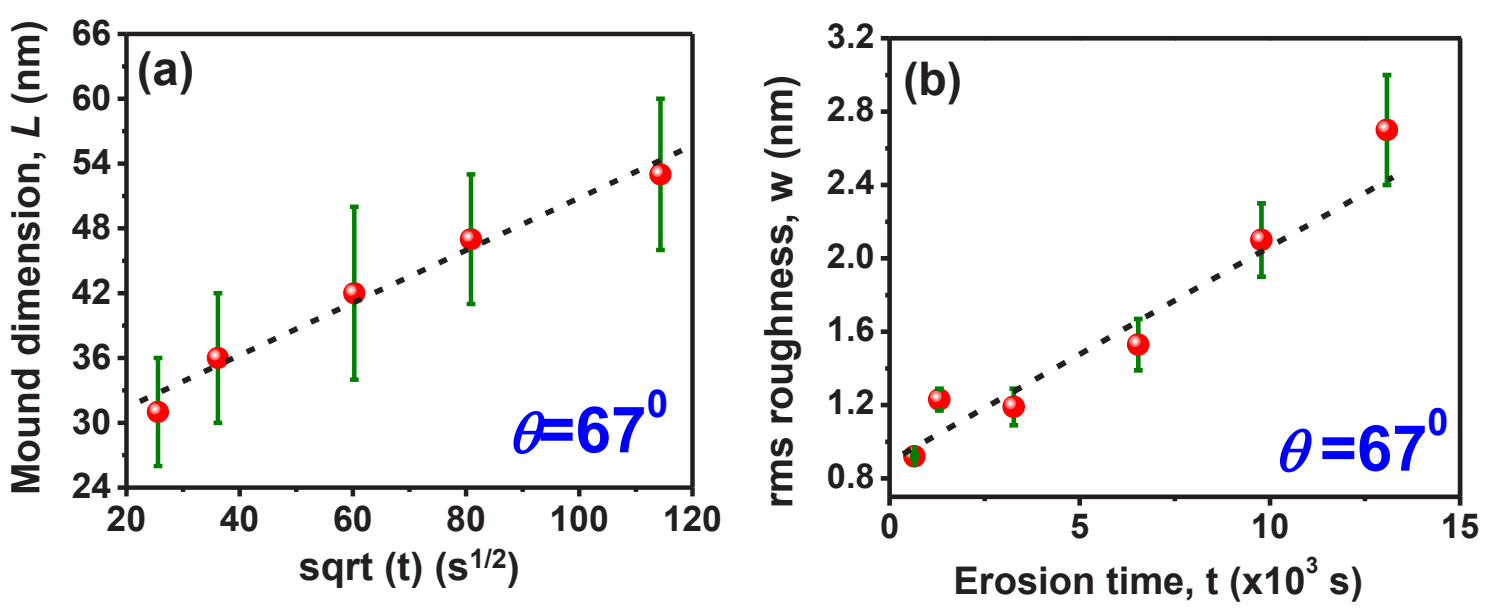

Basu et al.

Figure 6 


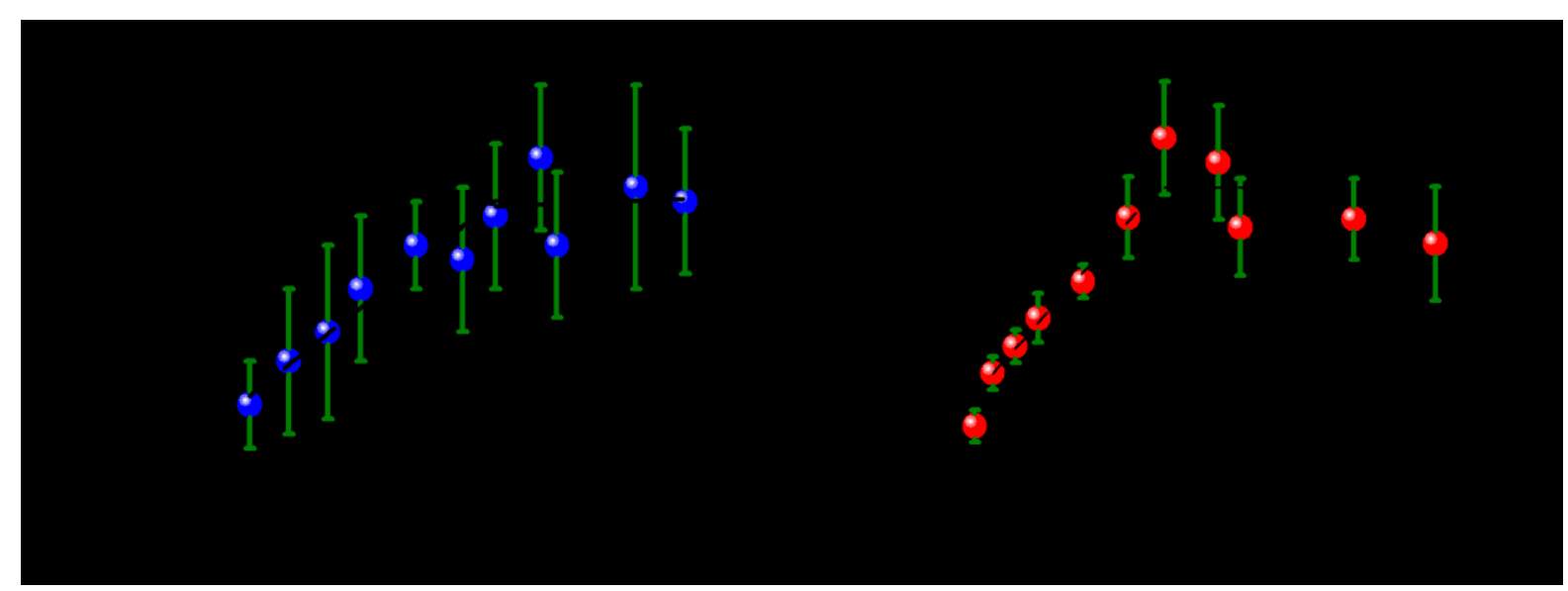

Basu et al.

Figure 7 

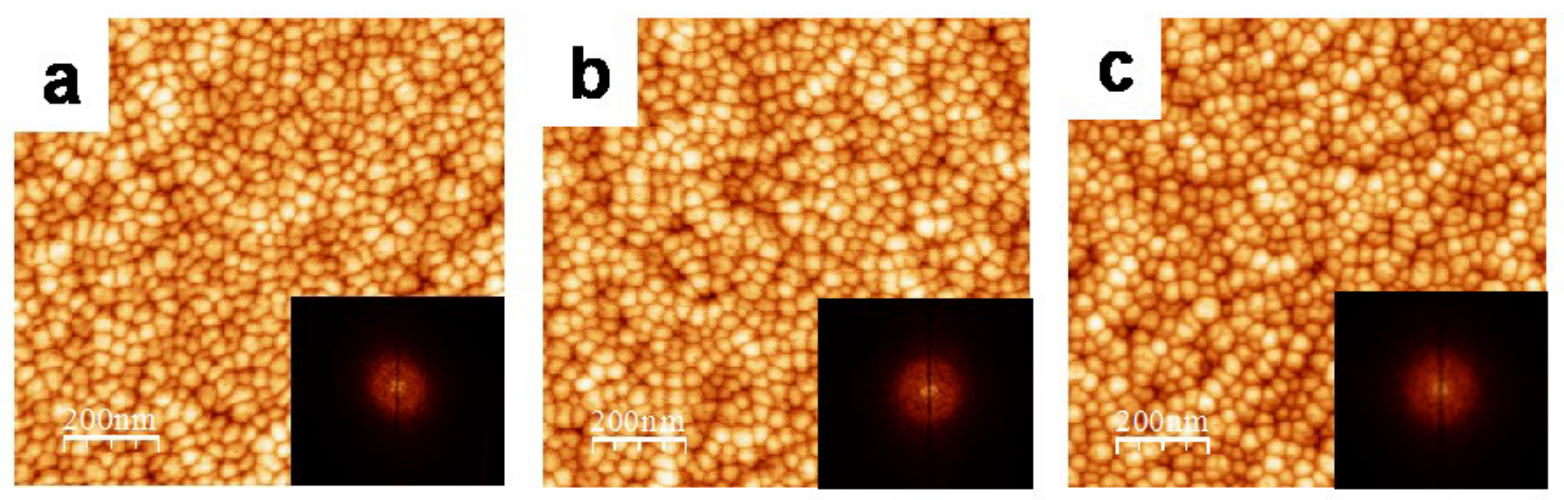

\section{Basu et al.}

Figure 8 


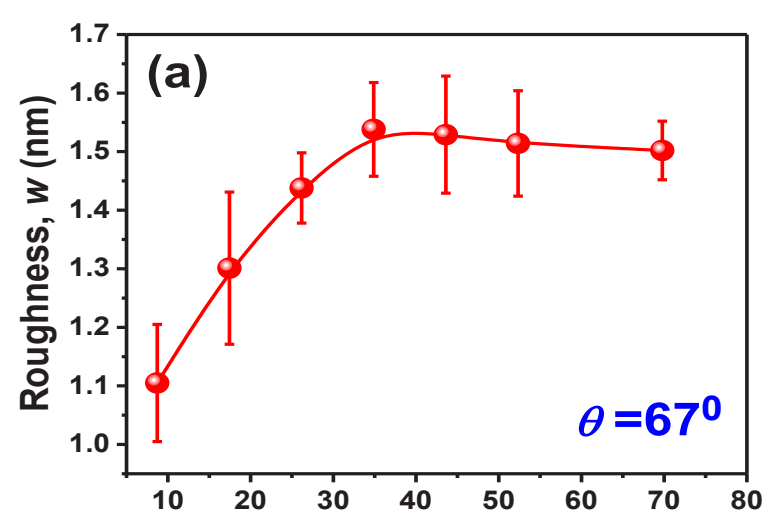

Rotational speed, ' $\omega$ ' (X 10 $\left.10^{-3} \mathrm{rad} / \mathrm{s}\right)$

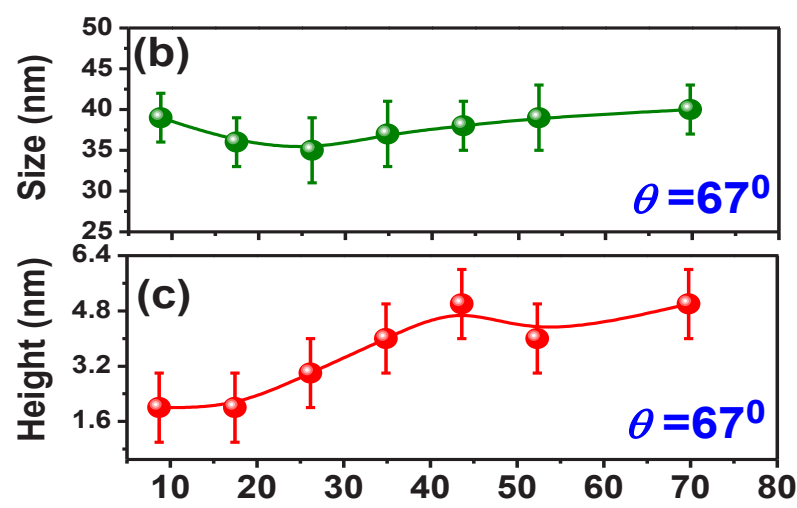

Rotational speed, ' $\omega$ ' (X 10 $\left.10^{-3} \mathrm{rad} / \mathrm{s}\right)$

Basu et al.

Figure 9 

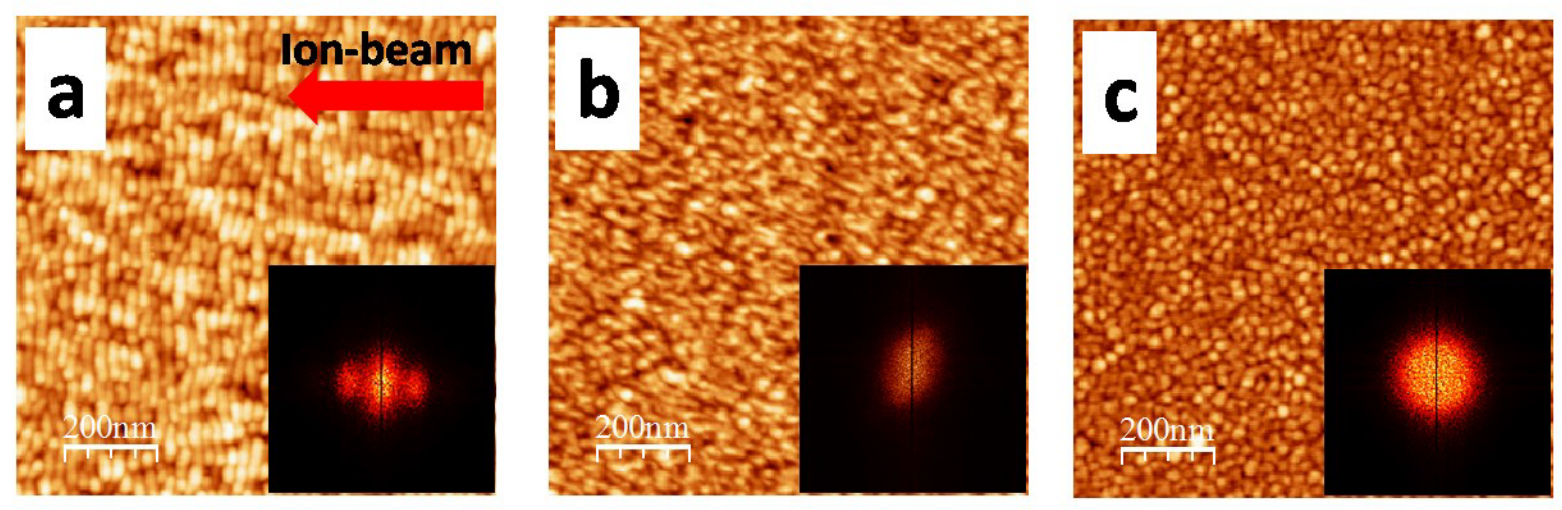

Basu et al.

Figure 10 


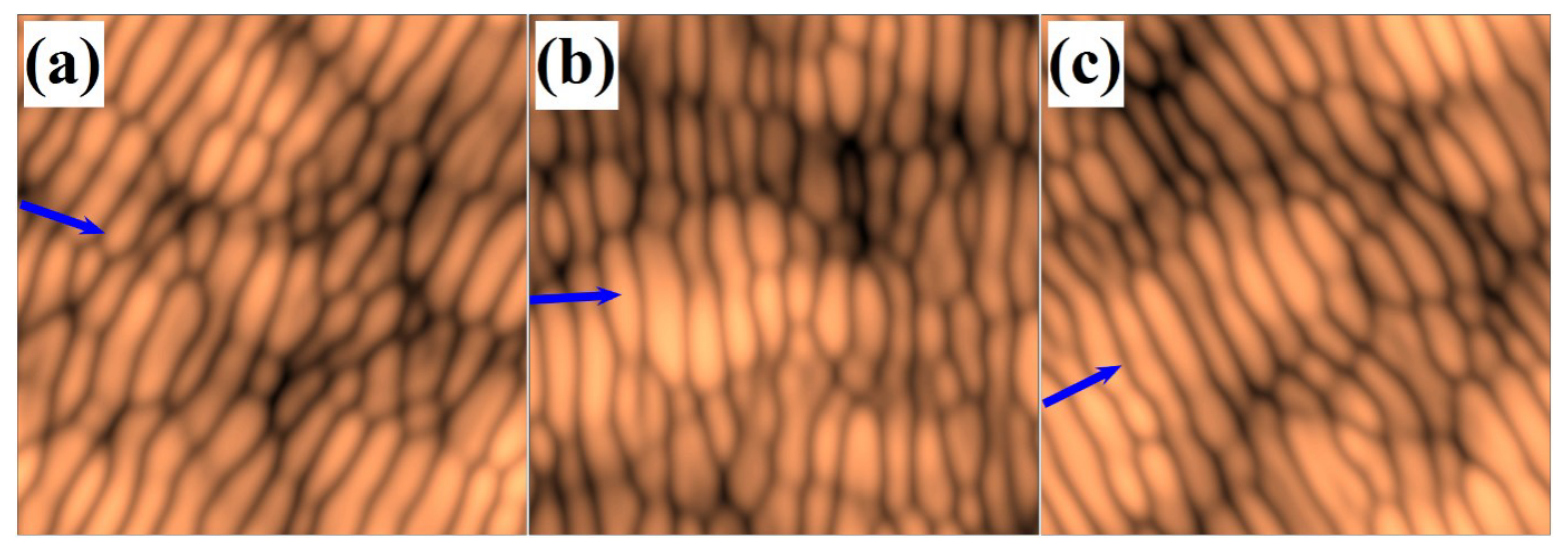

Basu et al.

Figure 11 


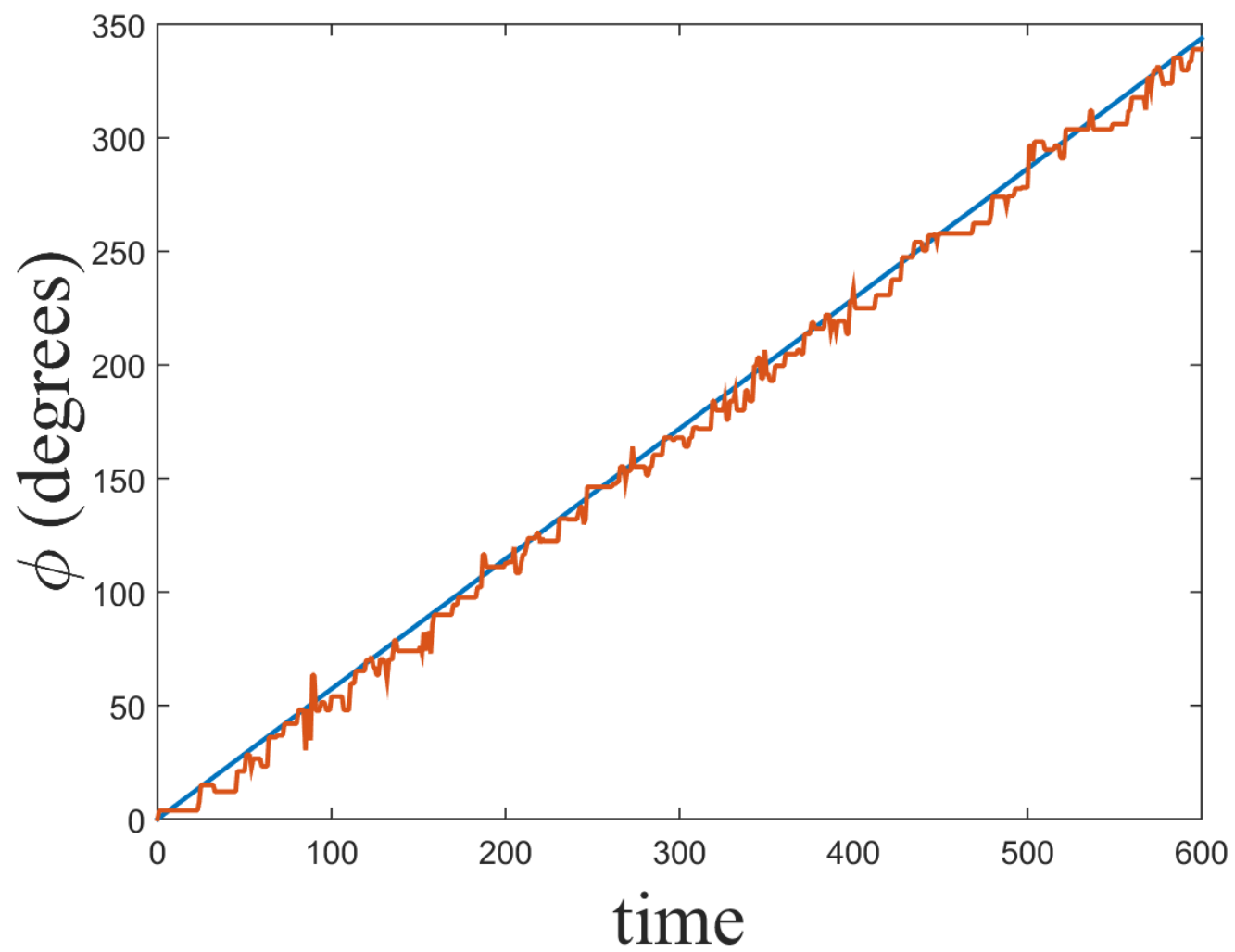

Basu et al.

Figure 12 


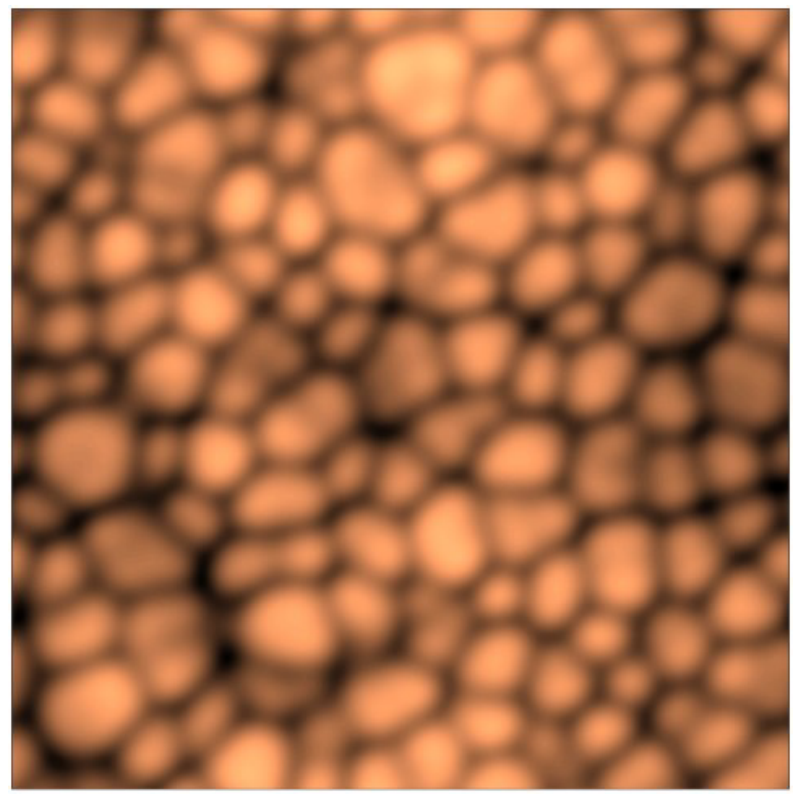

Basu et al.

Figure 13 